\title{
DIE BEDEUTUNG DER HANDWERKERRECHNUNGEN UND BIERDEPUTATE FÜR DEN OPERNBETRIEB DES JARMERITZER SCHLOSSTHEATERS IN DER ERSTEN HÄLFTE DES 18. JAHRHUNDERTS
}

Das Musikleben in Jarmeritz in der ersten Hälfte des 18. Jahrhunderts wurde schon von vielen Forschern aus verschiedenen Aspekten untersucht. Bis jetzt fehlte aber eine komplexere Betrachtung einigen Fragen der Musikpflege der musik-dramatischen Werken und des Theaterbetriebes an sich. Unbeachtet blieb dabei vor allem die theatrologische Ansicht. ${ }^{1}$ Deswegen soll diese Studie die bisherigen Erkenntnisse über die Gestalt des Jarmeritzer Theatersaals und über die Frequenz der dortigen Aufführungen erweitern und somit eine Grundlage für weitere theatrologische Forschung darstellen.

Der Bau des Schlosstheaters, das sich zusammen mit dem Ballhaus im Ostflügel des Schlosses befindet, wurde 1719 begonnen; der Ballspielsaal befand sich im Untergeschoss, der Theatersaal im ersten Stock. Er ist in den Quellen oft als „opera hau $\beta$ “ genannt. Überwiegend wird jedoch von einem comoedi-hau $\beta$ gesprochen, womit entweder das Theatersaal, das Ballspielsaal oder beide Säle

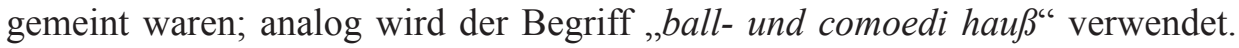
Die Bezeichnung „Theatrum“ wurde für die Bühne im Theatersaal benutzt. ${ }^{2}$ Der Theatersaal war mit seinem Ausmaß von 12,6 $\times 14,5$ Metern nicht sehr groß ${ }^{3}$ und war mit zwei Eingängen aus dem Vorraum zugänglich, in dem zwei Treppen mündeten. ${ }^{4}$ Im Zuschauerraum befanden sich mehrere Logen (die genaue Zahl ist unbekannt).

1 Erster Schritt in dieser Richtung präsentiert der Artikel von SPÁČILOVÁ, Jana. Odraz scénografie jaroměřického zámeckého divadla v libretech oper provedených zde v letech 17261743. In Hrabě Johann Adam Questenberg (1678-1752) - mecenáš a iniciátor hudebniho děni na Moravěv vobě baroka. Jana Perutková - Jiří K. Kroupa (hgg.). Clavibus unitis, 2014, Jg. 3, S. 135-144. Für die theaterwissenschaftliche Konsultation bedanke ich mich sehr bei Margita Havlíčková. Cfr. SPÁČILOVÁ, Jana. Operní divadla na Moravě v 1. polovině 18. století - současný stav vědomostí a perspektivy dalšího výzkumu. Opus musicum, 2011, Jg. 43, Nr. 6, S. 38-54. 
Im Mai 1722 wurde das „Theatrum Model“ in Wien eingepackt und nach Jarmeritz transportiert. ${ }^{5} \mathrm{Da}$ das Modell in Wien entstanden ist, kann angenommen werden, dass es zumindest unter der Beaufsichtigung Giuseppe Galli Bibienas errichtet wurde, oder sogar von ihm selbst. Der kaiserliche Theaterarchitekt Giuseppe Galli Bibiena (1696-1757) war Mitglied der berühmten Familie italienischer Theaterarchitekten und Bühnenbildner des 17. und 18. Jahrhunderts, die in verschiedenen bedeutenden italienischen und mitteleuropäischen Zentren wirkte. Giuseppe Galli Bibiena beeinflusste mit seiner Auffassung der Perspektive viele damalige Künstler und galt als der vorbildhafte Bühnenbildner nicht nur zu seinen Lebzeiten, sondern bis zum Ende des 18. Jahrhunderts. Er war mit Grafen Questenberg im regen Kontakt und hat für das Jarmeritzer Theater Bühnenbildentwürfe verfertigt. ${ }^{6}$ Eine bis jetzt wenig bekannte Tatsache ist, dass auch zwei talentierte Untertanen Questenbergs in Bibienas Werkstatt tätig und an den Saisonarbeiten beteiligt waren, nämlich die Maler Ignaz Buczek (Buček) und Joseph Schetinsky (Šetínský). Der Verbindung der Jarmeritzer Künstler und Bibienas Werkstatt soll weitere Forschung gewidmet sein.

Für die erstbelegte Aufführung im Jarmeritzer Schlosstheater wurde bis vor Kurzem eine näher nicht bestimmte Oper gehalten, die im November 1723 bei der Gelegenheit der Rückreise des Kaiserhofs aus Prag, wo Kaiser Karl VI. zum böhmischen König gekrönt war, im Jarmeritz gespielt wurde. Erst die sorgfältige Untersuchung von Rechnungen konnte nachweisen, dass erste Produktionen bereits im Jahre 1722 begonnen haben.

Die Rechnung vom 31. Dezember 1722 aus dem Zeitraum 5. Mai-19. Dezember 1722 für die Arbeit der Handwerker (Zimmermannleuthe) belegt die Vollendung des Theatersaals. ${ }^{7}$ Aus dem Jahre 1722 ist auch eine Rechnung des Jarmeritzer Färbers Matthias Kunst erhalten; er hat den Theatervorhang „,itron farb“ gemalt. ${ }^{8}$

ky ke katalogu praci Jakuba Prantauera (1660-1726). Brno, 1972. Dissertationsarbeit, Universita J. E. Purkyně, Filosofická fakulta, Seminář hudební vědy, s. 43-44.

5 Moravský zemský archiv (Mährisches Landesarchiv, weiter nur MZA), Fonds F 460, Kart. 2425, Inv. Nr. 9732, fol. 139v.

6 Die unlängst geglückte Auffindung und Identifizierung eines im Theatermuseum in Wien befindlichen Konvoluts von Bühnenbildentwürfen für das Jarmeritzer Schlosstheater stellt eine außerordentliche Bereicherung unserer bisherigen Kenntnisse über die Geschichte dieses Theaters dar. Die Bedeutung dieser neu entdeckten Entwürfe ist ganz besonders, denn sie sind die einzigen ikonographischen Belege für Opernaufführungen in mährischen Schlössern und Städten während der ersten Hälfte des 18. Jahrhunderts. Und nicht nur das: Vergleichbare Entwürfe in dieser Qualität und Menge sind nicht einmal für die bedeutendsten Opernbühnen dieser Zeit erhalten. Für mehr dazu siehe gerade erarbeitete Publikation von Jana Perutková, Jana Spáčilová, Andrea Sommer-Mathis und Martina Frank.

7 Die gesamte Summe war 166 fl. 58 kr. 2 pf. MZA, Fonds F 459, Kart. 680, Inv. Nr. 2493, fol. 237r-238r.

8 FIDLER, Petr. Příspěvky ke slovníku umělců a řemeslníků na Moravě v 17. a 18. století II. Sborník prací Filozofické fakulty brněnské univerzity, Reihe F, 1975-1976, Jg. 24-25, Nr. 19-20, S. 132. Für die Beratung bezüglich des Schlosstheaters bedanke ich mich herzlich bei Petr Fidler. 
Zeitgleich wurde auch das Bühnenportal verfertigt. In der mit 31. Oktober 1722 datierten Rechnung findet sich auch eine wichtige Eintragung für Trinkgeld für ,zimmerleüthen und tischlern, so bey der producirten opera arbeithen geholffen".9 Daraus geht hervor, dass die erste Opernaufführung im Herbst 1722 stattgefunden hat.

Sehr wertvoll ist die Rechnung für Tischlerarbeit in „opera hau $\beta^{\text {“ }}$ aus dem Jahr 1723, die „16 so genannte leviten stuhl worauf die Musici sitzen“ verzeichnet. Aufgrund der Kenntnisse über Orchesterbesetzungen bei den Opernaufführungen in der ersten Hälfte des 18. Jahrhundert ${ }^{10}$ können wir annehmen, dass das Questenbergsche Orchester am Anfang des regelmäßigen Opernbetriebes etwa aus 6 Violinen, 2 Violen, 2 Violoncelli, Violone, 2 Oboen, 1 Fagott, 1 Laute bzw. Theorba und einem Cembalo bestanden hat. ${ }^{11}$ Das Cembalo wurde laut der Rechnung des Prager Orgelmachers Adalbert Georg Dworsky vor der Aufführung vorbereitet, er hat in dieser Zeit auch ein Positiv aus seiner Werkstatt nach Jarmeritz - wahrscheinlich für die Loretto-Kappelle in Servitenkloster - gebracht. ${ }^{12}$

Als Theatermahler war in der Zeit der Znaimer Maler und Verwalter von Znaimer Haus Questenbergs, Franz Anton Findt, tätig. Für 1722 belegen die Rechnungen, dass er für ein Portal und zwei szenische Dekorationen bezahlt wurde..$^{13}$

Auch für die Feststellung des Titels und des Autors von der gesellschaftlich wichtigen Aufführung, die unter Teilnahme von einem Teil des kaiserlichen Hofstaates stattfand, war die Rechnung von Tischler Stöhring entscheidend. Wie schon erwähnt, war der Titel und Komponist des Werkes bis jetzt unbekannt. Zur Verfügung stand nur die Hypothese, dass es um eine nicht näher bekannte Oper von Wiener Hofkomponist Francesco Bartolomeo Conti ging, weil von ihm ein Werk in Jarmeritz im Juni 1723 abgeschrieben wurde. Im Brief des Jarmeritzer Verwalters Johann Joseph Stampa an den Grafen Questenberg vom 23. April war nämlich die Name vom diesen Komponisten genannt: „Der Both hat auch eine opera mitzubringen, welche der Jacob Rathauser $[=$ der Ratsherr und Organist in Jarmeritzer St. Margarethen-Kirche Jacob Mitscha] ehistens zu copieren, undt längstens zu Endt May wieder anhero zu schicken hat, dann H. Conti, der Sie

9 MZA, Fonds F 459, Kart. 680, Inv. Nr. 2493, fol. 128r.

10 Verschiedene Schriften von Johann Mattheson, Johann Joachim Quantz u.a.

11 Die Rechnung in MZA, Fonds F 459, Kart. 681, Inv. Nr. 2494, fol. 366v.

12 „Bei zweymahliger beküll. [= Bekielung, Montage der Federkiele] und stimmung des Instruments in opera hau $\beta$, wormit jedes mahl 1/2 tag zugebracht". Es handelte sich also um ein zweimanualiges Cembalo. MZA, Fonds F 459, Kart. 681, Inv. Nr. 2494, fol. 252r.

13 Die Quittung für „,das Portal contrahiertenmasßen gr.[oß]“ und „zwey Veränderungen Bestehend in 44 Thallarn [sog. „telari-Bühne“, d. h. drehbare dreiseitige Prismen nach dem Vorbild der antiken Periakten] und gartten postamentern [= Sockel]“ wurde über $102 \mathrm{fl}$. ausgestellt. Questenberg hat persönlich am 25. Oktober 1722 die Auszahlung von 82 fl. persönlich notiert. MZA, Fonds F 459, Kart. 680, Inv. Nr. 2493, nicht foliiert. Diese Auszahlung ist bestätigt ibid., fol. 39v: „Lauth ausziegl den 25 Octob: dem Frantz Antoni findt Burgrl: Mahler in Znaimb einige Bey dem Neuen Theatro gemachte arbeith Bezahlt 82 fl." 
componirt, solche Umb selbige Zeith wider haben will." Der Graf hat die Abschrift einige Tage später urgiert. ${ }^{14}$

Tischler Matthias Stöhring in seiner Rechnung aus 1723 erwähnt, dass er ,zur opera einen Thron vor die Könige Archelao und Sinopio" gemacht hat. Aus allen oben genannten Informationen geht hervor, dass es sich um die Tragicommedia Archelao re di Cappadocia von Francesco Bartolomeo Conti nach einem Libretto von Pietro Pariati handelte; das Werk wurde am Kaiserhof im Karneval 1722 uraufgeführt. ${ }^{15}$ In Jarmeritz war also am 17. November 1723 vor vielen hohen Aristokraten die Oper gespielt, die ein Jahr vorher in Wien erklang.

Graf Questenberg war aber offensichtlich mit dem Theater nicht völlig zufrieden, er war übrigens - wie bekannt - ein großer Perfektionist. Die Arbeiten im Theater- und im Ballspielsaal wurden deswegen noch im Jahre 1724 fortgesetzt, wie die Rechnungen für die Maurer-, Tischler- und Malerarbeiten, den Ankauf

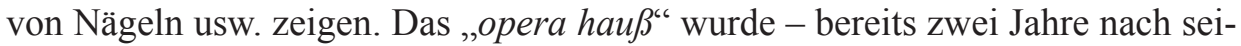
ner Fertigstellung - modernisiert. Das ,alte Theatrum “ [= Bühne] wurde nach Wien abtransportiert und in Jarmeritz ein neues installiert. Am 23. Juli 1724 hat der Wiener Theatertischler Johannes Patzelt, der für die Realisation der Bühnenbildentwürfe und für Maschinerie in Jaromeritzer Schlosstheater sehr wichtige Person war, ,wegen Aufsetzung des neuen Theatri" 20 fl. erhalten. ${ }^{16}$ Gleichzeitig ging der Abbau der alten Bühne voran, wie die ausgestellte Rechnung für die

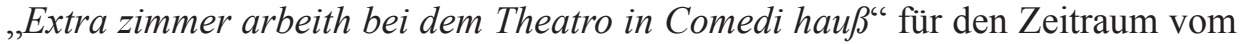
10. bis zum 15. Juli 1724 belegt. ${ }^{17}$

In derselben Zeit hat Matthias Stöhring zwei Rechnungen ausgestellt; er hat „den gemahlenen gartten in das alte Theatrum gemacht, wie auch blindtrahm [= einen Teil der Seitenkulisse, auf der die Dekorationen befestigt waren], und gesimser darzu, worüber 13 Tag zugebracht" und „das von wien überbrachte neue Theatrum eingericht, die stellen zum lampeln neu gemacht, worzu die gesellen 60 Tag zugebracht“ haben; dazu gehörte auch die „Außbeßerung des Theatri ${ }^{\prime \prime} .{ }^{18}$ Den von Stöhring erwähnten Gartenprospekt hat ein Jahr danach Franz Anton Findt neu ausgemalt. ${ }^{19} \mathrm{Im}$ Jahre 1724 wurden für das Theater auch 124 Lampen besorgt. ${ }^{20}$

14 MZA, Fonds G 436, Kart. 763, Inv. Nr. 6185, fol. 8r, 13 r.

15 SARTORI, Claudio. I libretti italiani a stampa dalle origini al 1800: Catalogo analitico con 16 indici. Cuneo: Bertola \& Locatelli, 1990-1994, Nr. 2386.

16 MZA, Fonds F 459, Kart. 682, Inv. Nr. 2494, fol. 22r.

17 Rechnung des Jarmeritzer Zimmermeisters Veit Lindtner. MZA, Fonds F 459, Kart. 682, Inv. Nr. 2494, fol. 136r.

18 MZA, Fonds F 459, Kart. 682, Inv. Nr. 2494, fol. 280r.

19 Rechnung aus 1725 u. a. für „Garten-Prospect sambt denen Scenen“, MZA, Fonds F 459, Kart. 683, Inv. Nr. 2496, fol. 375r. Für den „neu gemahlten Garten auf das Theatrum“ hat Jarmeritzer Schneider Johann Tvrdoczek im Jahre 1724 ,leinwand" fakturiert, er hat auch laut der Rechnung „,ie Scenen ausgebessert“. MZA, Fonds F 459, Kart. 682, Inv. Nr. 2494, fol. 278r.

20 MZA, Fonds F 459, Kart. 682, Inv.Nr. 2494, fol. 283r. Die Maurerarbeiten (121 fl.) in MZA, Fonds F 459, Kart. 683, Inv. Nr. 2495, fol. 40r. 
Im Jahre 1730 war Questenberg wahrscheinlich wieder mit dem Problem der Beleuchtung des Theaters beschäftigt, er suchte bei seinem Hofmeister Georg Adam Hoffmann Rat, an den er am 1. Juli 1730 schrieb: „Die lichter zur opera seynd meiner zeit niemahls auf 55. Gd.[Gulden], obschon alle illumina[ti]on erfordert worden, hinaufgeloffen: welche sachen alle eine tägliche inspection vonnothen hetten, damit ein, und anderen unrichtigkeiten vorgebogen werden könnte. “21

Im Jahre 1731 (also neun Jahre, nachdem der Theaterbetrieb in Jarmeritz aufgenommen worden war), wurden die Opernproduktionen wegen der Arbeiten an einem neuen Dach offensichtlich teilweise in den Ballspielsaal übertragen, wo man das so genannte, ffliehende Theatrum“" d. h. eine zerlegbare Holzbühne, verwenden konnte. ${ }^{22}$ Diese hat Questenberg wahrscheinlich auch in seinem Wiener Palais benutzt. Bei dieser Gelegenheit wurden vor allem an den hölzernen Bestandteilen im Theater einige Renovierungen vorgenommen. Der Bildhauer Caspar Ober hat 1732 nach seinem eigenen Entwurf auch zwei ,frontispicia“, kaschierte Löwenköpfe (,wappen lewen“), „Pasen und brust stucken“ für das Theater für $70 \mathrm{fl}$. verfertigt. ${ }^{23} \mathrm{Am} 12$. September desselben Jahres berichtete der Verwalter Widman dem Grafen: „an Verfertigung deren Scenen im Theatro wird

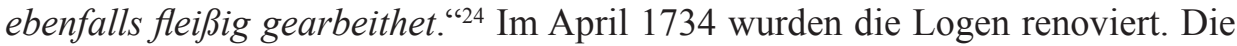
Arbeiten waren im Dezember 1734 mit den Stuckaturen und Vergoldungen abgeschlossen, rechtzeitig vor der Aufführung einer Serenata zum Namenstag des Grafen (Der glorreiche Nahmen Adami von Franz Anton Mitscha), der aus diesem Grund auf die Vollendung der Arbeiten sehr gedrängt hat. Für die Fertigstellung der neuen Ausgestaltung des Theaters war wahrscheinlich vor allem der italienische Maler und Theaterarchitekt Giovanni Pellizuoli verantwortlich.

Nach 1734 wurden noch kleinere Arbeiten an der Maschinerie unternommen, spätestens bis 1738 war ein „Flugwerck“ hergestellt. Im Jahre 1739 wurde die Bühne dem Vorbild des Theaters in Holešov/Holleschau angepasst, wie es eine Instruktion des Grafen belegt: „Hoffe ich, daß der Maurer- undt Zimmermeister mein nach dem Holleschauer Theatro recht richten werden, damit nicht allein die Szenen geschwindt und leicht geschoben, eine vor der andern recht gesehen werde, sondern auch zwischen ein jede Scene genugsamber Platz vor die actores zum herausgehen seye. Auch die Waltzen mit welchen die Szenen gezogen werden, so

21 Die Briefe Hoffmanns befinden sich in MZA, Fonds G 436, Kartons 747-748, Inv. Nr. 6133.

22 Brief des Grafen an Schloßverwalter Haussner aus 18. Juli 1731: ,[...] daß von ihm [= Zimmermeister] gemachten modely das fliehende theatrio balt zu standt bringen, damit das Ballhaus, wann keine commedi oder opera gespielet wirdt, könne zum spielen gebraucht werden." MZA, Fonds G 436, Kart. 765, Inv. Nr. 6186, fol. 389r. Es handelte sich um so genannte ,portatilia“, dazu z. B. ROHR, Julius Bernhard von. Einleitung zur Ceremoniel-Wissenschafft der Privat-Personen. Berlin: Rüdiger, 1728, S. 798-799.

23 Passe (aus dem Französischen passement) - Borte, Tresse. MZA, Fonds F 459, Kart. 688, Inv. Nr. 2503, fol. 41r. Sein Honorar hat er erst im Jahre 1735 erhalten, siehe FIDLER, Petr. Př́spěvky ke slovníku umělců a řemeslníků na Moravě 17. a 18. století I. Sborník prací Filozofické fakulty brněnské univerzity, Reihe F, 1974, Jg. 23, Nr. 18, S. 87. 
leicht als zu Holleschau durch zwei Personen gedrehet werden mögen.“25 Diese Arbeiten im Theater waren bereits am Beginn des Jahres 1739 vollendet.

Der Tischler Matthias Stöhring war für Questenberg zumindest in den Jahren 1713-1741 tätig. Es sei erwähnt, dass Stöhring nicht nur für Schloss und Theater gearbeitet hat, sondern auch gelegentlich Kulissen für das Heilige Grab in der St. Margarethen-Kirche verfertigte. Die Arbeit für die Schlossbühne hat er, wie schon angegeben, von Wiener Theatertischler Johannes Patzelt gelernt. Als Aushilfe für Patzelt oder Stöhring wird in den Quellen oft auch der Zimmermann

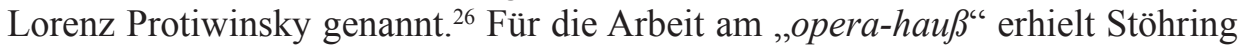
im Jahre 1723 sogar mehr als Patzelt, nämlich hohe Summe 210 fl. 49 kr. 2 pf. ${ }^{27}$ Für die Zeit von 5. Juli bis Ende Dezember 1734 bekam er für seine Arbeit im „comedi hauß“ 196 fl. 25 kr. ${ }^{28}$ Er war auch bei den einzelnen Aufführungen als Maschinist anwesend, wofür er ein Deputat (Bier) erhielt, was unten ausführlicher behandeln wird. Seine erste Tätigkeit in diesem Sinne dokumentiert eine Rechnung aus dem Jahre 1724: „auf 4 Operen alles zugericht, und regirt 5 stundt lang mit 4 gesellen, undt 2 buben dazu gehabt" ${ }^{.29}$ Daraus kann man schließen, dass am Beginn des Opernbetriebes in Jarmeritz die Maschinerie im Theater etwa von fünf Männern und zwei Knaben bedient wurde.

Sehr wertvoll ist die Bier-Rechnung des Tischlers Stöhring aus dem Jahre 1736 für die Maschinisten und die Komparserie (jeder hat eine Maß Bier pro Aufführung erhalten). Diese Quelle bringt nicht nur die genauen Daten von Aufführungen und über gespielten Gattungen, sondern auch über die Gesamtzahl des Hilfspersonals. Daraus ergibt sich, dass an den Aufführungen ständig neun Tischler beteiligt waren; die Anzahl der Zimmerleute bewegte sich zwischen 4-9

25 Brief des Grafen an den Verwalter Widmann vom 28. Jänner 1739. MZA, Fonds G 436, Kart. 767, Inv. Nr. 6188, fol. 316r.

26 Im Jahre des Umbaus des Ostflügels des Schlosses (1734), wo Ballspielsaal und der Theater-

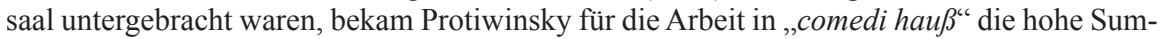
me von 1.220 fl. 42 kr. 1 pf. MZA, Fonds F 459, Kart. 689, Inv.Nr. 2504, fol. 43r. Lediglich im Jahre 1734 kommt in den Jarmeritzer Rechnungen ein Wiener Tischler Christian Holtz vor, der im „camedi hauß“ arbeitete. Er erhielt für 153 Tage 43 fl. 21 kr. MZA, Fonds F 459, Kart. 689, Inv. Nr. 2504, fol. 45r.

27 MZA, Fonds F 459, Kart. 681, Inv. Nr. 2494, fol. 40r.

28 MZA, Fonds F 459, Kart. 689, Inv. Nr. 2504, nicht foliiert. Für das ganze Jahr 1734 erhielt er für die Arbeit im „comedi hauß“" insgesamt 281 fl. 42 kr., Patzelt dann 29 fl. 15 kr. (MZA, Fonds F 459, Kart. 689, Inv.Nr. 2505, fol. 56r.). Sein Honorar umfasste jedoch nicht nur die Herstellung der Bühnenbilder, sondern auch verschiedene andere Arbeiten im Theater oder auch im Schloss. In Mai 1735 hat u.a. auch ,neue waagen“ für das Theater angefertigt (MZA, Fonds F 459, Kart. 690, Inv. Nr. 2506, fol. 368r.). Leider sind seine Arbeiten in den Rechnungen meistens nicht spezifiziert (oft finden wir nur die Angabe, für verschiedene Tischlerarbeith"); jedenfalls handelte es sich in einigen Jahren um hohe Summen, z. B. 1733 (im April 1734 wurden ihm für das ganze Jahr $1733401 \mathrm{fl}$. $15 \mathrm{kr}$. bezahlt), für 1735 (605 fl.), 1736 (445 fl.) oder 1737 (1738 wurden ihm 470 fl. bezahlt). MZA, Fonds F 459, Kart. 689, Inv. Nr. 2504, fol. 95v.; ibid., Kart. 691, Inv. Nr. 2506, fol. 43v.; ibid., Kart. 691, Inv. Nr. 2506, fol. 83r; ibid., Kart. 693, Inv. Nr. 2508 fol. 21r.

MZA, Fonds F 459, Kart. 682, Inv. Nr. 2494, fol. 221r. 
Personen, gelegentlich kamen 3-4, „Buben“ dazu. Die Anzahl der Handwerker, die vor allem für die Maschinerie verantwortlich waren, ist also im Vergleich zum Jahr 1724 gestiegen. Die Verwendung der Komparserie (Stabene) war von der Gattung abhängig: Bei den Komödien waren es üblicherweise 2-6, während bei den Opern 22-33 Personen, einmal sogar 46 [sic!] Komparsen aufgetreten sind. $\mathrm{Zu}$ diesem Personal haben auch noch 2 ,gerichts diener" gehört.

Stöhring hat oft sehr ausführliche Berichte in seinen Rechnungen formuliert, aus denen geht hervor, dass er die wichtigste Person für Bühnenausstattung war. Er hat z. B. im Jahre 1725 u. a. folgende die Bühne betreffende Arbeiten verrechnet: ,[...] auf dem Theatro neuen prosspect, undt den alten zurück gericht, eine große Truchen [= Truhe], eine Weldtkugel, ${ }^{30}$ item 2. tisch., ein Spiegl, 2. sessel, 2. spielhüttern, 3. wägen, worauf die Kinder hierunter gefahren, 4. Trüchl vor die Täntzer, ein banck, 5. pritschen, 4. helleparthen, undt was auf dem Theatro sonst gebrochen geweßen, zurecht gemacht, auch vor dem feüerwerckher etwelche Sachen verfertiget ". ${ }^{31}$ Aus dieser Rechnung ist ersichtlich, dass das Jarmeritzer Theater bereits in der Mitte der 1720er Jahre gut ausgestattet war.

Einige von seinen Rechnungen für seine Theaterarbeiten bilden ein wichtiges Zeugnis für die Frequenz der von Questenberg in Jarmeritz veranstalteten Theateraufführungen. Er hat im Jahre 1725 seine Arbeit für 13 Komödien, ${ }^{32} 1732$ für insgesamt 40 Opern und Komödien, ${ }^{33}$ zwei Jahre danach für 18 Opern und 16 Komödien, also für 34 Aufführungen verrechnet. Ein weiteres Beispiel bildet die Quelle aus dem Jahre 1736: Laut der erhaltenen Rechnung wurde im Jarmeritzer Schlosstheater vom Jänner bis März dieses Jahres nicht gespielt; während dieser Zeit hat sich wahrscheinlich der Graf in Brünn als Prinzipalkommissar beim mährischen Landtag in Brünn etabliert. Vom April bis Ende September 1736 wurden dennoch 16 Opern und 12 Komödien aufgeführt. Die große Anzahl ist überraschend, weil Questenberg zu dieser Zeit wegen seiner Funktion des Kommissars in Brünn vorwiegend anwesend sei. Die diesbezügliche Liste der Aufführungen bringt folgende Daten:

2. 4. Oper [wahrscheinlich Didone abbandonata von Leonardi Vinci],

3. 4. Komödie,

4. 4. Oper,

8. 4. Oper,

9. 4. Komödie,

30. 5. Komödie,

13. 6. Oper,

14. 6. Komödie,

17. 6. Oper,

30 Von „Globen“ im Theater sprechen auch andere Jarmeritzer Quellen.

31 MZA, Fonds F 459, Kart. 683, Inv. Nr. 2496, fol. 273v.

32 MZA, Fonds F 459, Kart. 683, Inv. Nr. 2496, fol. 272v.

33 MZA, Fonds F 459, Kart. 688, Inv. Nr. 2503, fol. 58r. 
18. 6. Komödie,

19. 6. Oper,

3. 7. Oper,

4. 7. Oper,

8. 7. Komödie,

10. 7. Oper,

11. 7. Oper,

12. 7. Komödie,

18. 7. Komödie,

19. 7. Oper,

20. 8. Komödie,

23. 8. Oper [extra Summe für die in der Komparserie mitwirkenden „12 Soldaten, so sich öfters exercieren"],

24. 8. musica navalis auf dem Fluss Jarmeritza, heute Rokytná [die Entlohnung für die „Schiffleuthen" u.a.],

25. 8. Komödie,

26. 8. Oper,

30. 8. Komödie,

8. 9. Oper,

9. 9. Komödie,

19. 9. Oper,

21. 9. Oper.

Neben Stöhrings Rechnungen sind auch noch andere Quellen dieses Fonds für die Frequenz der Jarmeritzer Aufführungen wertvoll, vor allem die vom Jarmeritzer Verwalter Ivo Joseph Widmann aufgestellten sog. „Bier Register“. Einige von diesen Registern beinhalten nämlich die Biermenge für die an den Aufführungen beteiligten Personen. So kann man einen Überblick über Aufführungsdaten gewinnen; manchmal auch über theatralischen Gattungen, die in Jarmeritz zu jener Zeit gespielt wurden. Ausnahmsweise ist auch die Anzahl des Hilfspersonals (Maschinisten usw.) belegt.

Die Kombination der Stöhrings Rechnung und des Bier-Registers aus dem Jahre 1738 bildet den Gesamtüberblick über theatralischen Gattungen und musikdramatischen Aufführungen, die in dieser Zeitspanne geübt und gespielt wurden. ${ }^{34}$ Eine Ergänzung stellt noch eine Rechnung des Malers Ignaz Buczek aus demselben Jahr dar, für die ,mahler arbeith bey der Illumination alß auch in dem

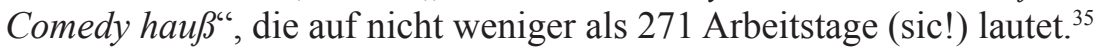

1. 1. Komödie,

2. 1. Oper,

34 MZA, Fonds F 459, Kart. 693, Inv. Nr. 2508, fol.88r. ibid., Inv. Nr. 2509, fol. 65r-v, Fol 88r-v.

35 Die Rechnung über die gesamte Summe von 76 fl. 47 kr. beinhaltet auch genaue Daten. MZA, Fonds F 459, Kart. 693, Inv.Nr. 2509, nicht foliiert. 
6. 1. Oper,

7. 4. Oper (Ostermontag),

26. 4. Oper,

27. 4. Komödie,

1. 5. Komödie,

3. 5. Oper,

4. 5. Komödie,

11. 5. Oper,

12. 5. Oper,

12. 6. Opernprobe,

13. 6. Serenata im Heckentheater [Domenico Natale Sarro: Il giudizio di Paride, Uraufführung],

14. 6. Komödie,

15. 6. neue Oper [Ignazio Maria Conti: Il delizioso ritiro scielto da Lucullo, console Romano, Uraufführung],

16. 6. Komödie,

17. 6. Komödie,

24. 6. Komödie [„Portsionell Comoedie“ $=$ Marionettentheater mit Porzellanpuppen ${ }^{36}$,

26. 6. Komödie [„Portsionell Comoedie“],

29. 6. Komödie [,Portsionell Comoedie“],

2. 7. „,öhmische opera“ [Franz Anton Mitscha: L'origine di Jaromeriz, Neuanstudierung],

3. 7. italienische Oper,

5. 7. Komödie,

15. 7. Oper,

16. 7. Komödie,

22. 7. Komödie,

21. 8. Komödie,

26. 8. Oper,

27. 8. Komödie,

28. 8. Komödie,

17. 9. Komödie,

21. 9. Komödie,

25. 9. Komödie,

28. 9. Oper,

29. 9. Komödie,

19. 10. Oper,

36 Das Inventar von Questenbergs Nachlass aus dem Jahre 1752 bringt die Information über ein „,kleines Theatrum“ mit „Boruezinelli figuren oder Marionetten“ (MZA, Zn. C2, Fonds: Tribunal - Nachlässe, Kart. 176, Sign. Q6, 1752, fol. 14v.). Es handelte sich um die Figuren aus der commedia dell'arte darstellende Puppen mit Porzellankopf und um hölzerne Marionetten. Das Theater mit den „Porzellanpuppen“ wurde im Jahre 1724 errichtet, es wurden dafür auch 12 Lampen angekauft (,12 lattern“ für „,borcenell theatrum“, MZA, Fonds F 459, Kart. 682, Inv. Nr. 2494, fol. 220r). Den Quellen zufolge war der Raum, wo dieses Theater gespielt wurde, die Sala terrena im Jarmeritzer Schloss (MZA, Fonds F 459, Kart. 682, Inv. Nr. 2494, fol. 129v). 
21. 10. Komödie,

23. 10. Oper,

4. 11. Oper,

11. 11. Komödie,

8. 12. Opernprobe,

16. 12. Opernprobe,

17. 12. böhmische „Kinder comedy" [von Kindern aufgeführt],

18. 12. Opernprobe,

21. 12. Opernprobe,

22. 12. Opernprobe,

26. 12. Opernprobe,

27. 12. Oper,

28. 12. Oper [Giuseppe Ferdinando Brivio: Demofoonte, Uraufführung],

29. 12. Komödie,

30. 12. Komödie,

31. 12. Komödie,

1. 1.1739 Oper,

4. 1. Komödie,

6. 1. Oper.

Es scheint, dass Graf Questenberg im Jahre 1738 seine Frau Maria Antonia, geb. von Kaunitz, aber auch seine Freunde unter dem mährischen Adel mit dieser hohen Anzahl von musik-theatralischen Produktionen tief beeindrucken wollte. Aus den zitierten Quellen geht hervor, dass in Jarmeritz in diesem Jahr (bis zum 6. Jänner 1739) 21 Opern (einschließlich der im Freien aufgeführten Serenata $I l$ giudizio di Paride) und 27 Komödien (inklusive der mit Marionetten gespielten Komödien und einer von Kindern aufgeführte Komödie), d. h. insgesamt 48 Vorstellungen stattgefunden haben. Auffällig ist auch, wie sorgfältig - mit sechs Proben auf der Bühne - die „Hochzeitsoper“ für Questenbergs Tochter Maria Carolina vorbereitet wurde. Es ging um das aufgrund Giuseppe Ferdinando Brivios Demofoonte zusammengestellte Pasticcio. Außer dieser Hochzeit im Dezember hat der Graf im April auch seine eigene, zweite Hochzeit gefeiert; der rege gesellschaftliche Betrieb erklärt wahrscheinlich das Übergewicht an Komödien.

Mit Hilfe der Jarmeritzer Hanwerkerrechnungen ist es anzunehmen, dass sich die Anzahl der Jarmeritzer Opernaufführungen nach und nach gesteigert hat. In der zweiten Hälfte der 1720er Jahre waren es etwa 15 Aufführungen, in den 1730er Jahren etwa 20. Man sieht auch, dass im Schlosstheater auch mehrere Reprisen verschiedener Werke stattgefunden haben. Meistens zweimal, aber auch dreimal im Jahr wurde eine neue Oper einstudiert, gewöhnlich im Herbst und gegen Ende des Jahres, oder auch im Frühling, bzw. unmittelbar nach Ostern. Dieser Zeitplan wurde selbstverständlich nicht immer konsequent eingehalten, aber die allgemeine Tendenz, zwei bis dreimal im Jahr ein neues Werk zu bringen, ist deutlich zu erkennen. Dazu kamen auch jährliche Aufführungen von Sepolcri und Huldigungskompositionen, vor allem für Namenstage bzw. Geburtstage des Grafen. 
Die Reprisen fanden nicht nur im Jahre ihrer Erstaufführungen statt, sondern sie wurden auch später wiederholt, oft im der Premiere darauffolgenden Jahr. Dies betrifft z. B. die 1737 und 1738 aufgeführte Merope von Riccardo Broschi, oder Johann Adolph Hasses Cajo Fabricio o sia Pirro (1734, 1735). Einige Werke wurden auch in einem größeren Zeitabstand wiederholt; so wurde z. B. Mitschas Oper L'origine di Jaromeriz in Moravia 1730 uraufgeführt und zumindest 1738 neu einstudiert. Die Anzahl an etwa 170 Veranstaltungen von musikdramatischen Werken, die auf Initiative von Graf Questenberg in seinem Zentralsitz in Jarmeritz veranstaltet wurden, kann sogar mit den einigen professionellen Opernstagioni verglichen werden.

Wie ersichtlich, nicht alle aus den erhaltenen Questenbergschen Quellen völlig ausgewertet wurden. Die interdisziplinäre Mitarbeit hinsichtlich des Jarmeritzer Schlosstheaters bringt hoffentlich weitere Erkenntnisse über dieses bemerkenswerte Phänomen, das nicht nur für Länder der böhmischen Krone, sondern auch für mitteleuropäische Operngeschichte interessant und bedeutungsvoll ist.

Jana Perutková (perutkov@phil.muni.cz), Ústav hudební vědy, Filozofická fakulta, Masarykova univerzita, Brno, CZ.

\section{ABSTRACT \\ THE IMPORTANCE OF WORKMANSHIP AND „BIERDEPUTATE“ ACCOUNTING DOCUMENTS FOR OPERATIC PERFORMANCES AT JAROMĚŘICE CASTLE THEATRE IN THE FIRST HALF OF THE $18^{\text {th }}$ CENTURY}

This study broadens knowledge about the appearance of Jaroměrice castle theatre during the reign of count Johann Adam von Questenberg and about the frequency of Jaroměřice theatre's performances as well. There is unambiguous evidence that the first performance was held at this theatre as early as 1722. Moreover, thanks to the accounting documents by Jaroměrice's joiner Matthias Stöhring it was discovered which opera was performed in front of the Imperial Court members in 1723. The title of that opera was Archelao re di Cappadocia by the composer Francesco Bartolomeo Conti. The mentioned work had been performed for the first time one year earlier for the Imperial Court in Vienna. The originally used stage was moved to Vienna in 1724 and was followed by an installation of a new modern stage in Jaromeríce. Within the presented study the further adjustments and reconstructions of the theatre hall are observed up to the year 1739 .

From Stöhring's accounts we know not only the number of dramatic performances but for some years, we are able to find out even particular dates and genres. We are also able to reveal valuable information about Jaroměrice theatre's machinery. In 1730s, there were always 9 joiners, $4-9$ carpenters and $3-4$ auxiliary boys taking part in each performance. This staff included usually $2-3$ "gerichts diener". The number of extras was variable according to the genre; for comedies there were mostly $4-6$ extras, for operas $22-33$. It is obvious that not all preserved questenbergian sources have been fully evaluated yet. Interdisciplinary cooperation in the problematic of the Jaroměrice castle theatre will bring further information about this remarkable phenomenon, which is interesting not only in the context of Bohemian lands, but also for history of opera in the Central Europe. 


\section{Key words}

Questenberg, Moravia, Jarmeritz, Theater, scenography, machinery, opera seria, 1st half of the 18th Century

\section{Bibliography}

\section{Sources}

MZA, Fonds F 460, Kart. 2425, Inv. Nr. 9732.

MZA, Fonds F 459, Kart. 680, Inv. Nr. 2493.

MZA, Fonds F 459, Kart. 681, Inv. Nr. 2494.

MZA, Fonds F 459, Kart. 682, Inv. Nr. 2494.

MZA, Fonds F 459, Kart. 683, Inv. Nr. 2495.

MZA, Fonds F 459, Kart. 688, Inv. Nr. 2503.

MZA, Fonds F 459, Kart. 689, Inv.Nr. 2504.

MZA, Fonds F 459, Kart. 689, Inv.Nr. 2505.

MZA, Fonds F 459, Kart. 690, Inv. Nr. 2506.

MZA, Fonds F 459, Kart. 691, Inv. Nr. 2506.

MZA, Fonds F 459, Kart. 693, Inv. Nr. 2508.

MZA, Fonds F 459, Kart. 693, Inv.Nr. 2509.

MZA, Zn. C2, Fonds: Tribunal - Nachlässe, Kart. 176, Sign. Q6, 1752.

MZA, Fonds G 436, Kart. 763, Inv. Nr. 6185.

MZA, Fonds G 436, Kart. 765, Inv. Nr. 6186.

MZA, Fonds G 436, Kart. 766, Inv. Nr. 6187.

MZA, Fonds G 436, Kart. 767, Inv. Nr. 6188.

MZA, Fonds G 436, Kartons 747-748, Inv. Nr. 6133.

\section{Literature}

FIDLER, Petr. Přestavba zámku v Jaroméřicich nad Rokytnou v letech 1709-1737. Přispěvky ke katalogu prací Jakuba Prantauera (1660-1726). Brno, 1972. Dissertationsarbeit, Universita J. E. Purkyně, Filosofická fakulta, Seminář hudební vědy.

FIDLER, Petr. Příspěvky ke slovníku umělců a řemeslníků na Moravě 17. a 18. století I. Sborník prací Filozofické fakulty brněnské univerzity, Reihe F, 1974, Jg. 23, Nr. 18, S. 78-91.

FIDLER, Petr. Př́spěvky ke slovníku umělců a řemeslníků na Moravě v 17. a 18. století II. Sborník praci Filozofické fakulty brněnské univerzity, Reihe F, 1975-1976, Jg. 24-25, Nr. 19-20, S. 127-137.

ROHR, Julius Bernhard von. Einleitung zur Ceremoniel-Wissenschafft der Privat-Personen. Berlin: Rüdiger, 1728.

SARTORI, Claudio. I libretti italiani a stampa dalle origini al 1800: Catalogo analitico con 16 indici. Cuneo: Bertola \& Locatelli, 1990-1994.

SPÁČILOVÁ, Jana. Operní divadla na Moravě v 1. polovině 18 . století - současný stav vědomostí a perspektivy dalšího výzkumu. Opus musicum, 2011, Jg. 43, Nr. 6, S. 38-54.

SPÁČILOVÁ, Jana. Odraz scénografie jaroměřického zámeckého divadla v libretech oper provedených zde v letech 1726-1743. In Hrabě Johann Adam Questenberg (1678-1752) - mecenáš a iniciátor hudebního dění na Moravě v době baroka. Jana Perutková - Jiří K. Kroupa (hgg.). Clavibus unitis, 2014, Jg. 3, S. 135-144. 